ARTICLE

\title{
A silicate dynamo in the early Earth
}

\author{
Lars Stixrude (1) ${ }^{1,2 \times}$, Roberto Scipioni ${ }^{2} \&$ Michael P. Desjarlais ${ }^{3}$
}

The Earth's magnetic field has operated for at least 3.4 billion years, yet how the ancient field was produced is still unknown. The core in the early Earth was surrounded by a molten silicate layer, a basal magma ocean that may have survived for more than one billion years. Here we use density functional theory-based molecular dynamics simulations to predict the electrical conductivity of silicate liquid at the conditions of the basal magma ocean: 100-140 GPa, and 4000-6000 K. We find that the electrical conductivity exceeds 10,000 S/m, more than 100 times that measured in silicate liquids at low pressure and temperature. The magnetic Reynolds number computed from our results exceeds the threshold for dynamo activity and the magnetic field strength is similar to that observed in the Archean paleomagnetic record. We therefore conclude that the Archean field was produced by the basal magma ocean.

\footnotetext{
${ }^{1}$ Department of Earth, Planetary, and Space Sciences, University of California, Los Angeles, CA 90095, USA. ${ }^{2}$ Department of Earth Sciences, University College London, London WC1E 6BT, UK. ${ }^{3}$ Pulsed Power Sciences Center, Sandia National Laboratories, Albuquerque, NM 87185, USA.

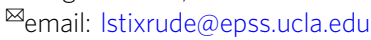


T he Archean magnetic field may have been essential for preserving the early atmosphere and hydrosphere ${ }^{1}$. The process by which the field is produced today: a dynamo hosted in the Earth's metallic iron-rich liquid outer core is thought to be difficult to sustain in the early Earth because the core could not cool sufficiently rapidly ${ }^{2-4}$. Alternative energy sources, including radioactive heat production in the core or exsolution of $\mathrm{Mg}$ from the cooling core, may not be sufficient to sustain an early dynamo.

Planetary dynamos in the solar system are hosted in a variety of materials including iron-rich liquid, such as in the Earth and other terrestrial planets, ionically conductive fluid ices, as in Uranus and Neptune, and metallic hydrogen as in Jupiter and Saturn ${ }^{5}$. A silicate dynamo is so far unknown. Magnetohydrodynamic simulations, laboratory experiments, and studies of planetary bodies that host magnetic fields show that dynamo activity requires a sufficiently large magnetic Reynolds number $R_{\mathrm{m}}=\mu_{0} v L \sigma>40$, where $\mu_{0}$ is the magnetic susceptibility, $v$ is the flow velocity, $L$ is the depth of the layer, and $\sigma$ is the electrical conductivity ${ }^{6,7}$. Using illustrative values, $R_{\mathrm{m}}=40(\mathrm{v} / 1 \mathrm{~cm} / \mathrm{s})(L / 300 \mathrm{~km})(\sigma / 10,000 \mathrm{~S} / \mathrm{m})$. Therefore, for a silicate dynamo to operate the electrical conductivity must exceed $10,000 \mathrm{~S} / \mathrm{m}$, more than 100 times higher than the highest values measured in silicate liquids at low pressure and temperature ${ }^{8,9}$, although less than typical metallic conductivity $\left(10^{6} \mathrm{~S} / \mathrm{m}\right.$ for the Earth's outer core).

Here we perform first principles molecular dynamics simulations of a silicate liquid that closely approximates the composition of the bulk silicate Earth and includes the six most abundant oxide components; the size of our simulation is 1129 atoms (see Methods). The simulations and the computation of the electrical conductivity closely follow our previous work on the $\mathrm{SiO}_{2}$ and $(\mathrm{Mg}, \mathrm{Fe}) \mathrm{O}$ systems ${ }^{10,11}$ (see Methods). Briefly, we compute the electronic contribution to the electrical conductivity with the Kubo-Greenwood formula ${ }^{12}$ and the ionic conductivity from the electric current autocorrelation function ${ }^{13}$. In order to investigate the effect of magnetism on our results, we consider non-spin-polarized and spin-polarized results with the latter characterized by high-spin moments on all the iron atoms. We combine our predictions of electrical conductivity with a model of the thermal evolution of the basal magma ocean to estimate the magnetic Reynolds number and the strength of the magnetic field generated by the silicate dynamo. We find that the magnetic Reynolds number exceeds 40 and that the field strength is in excellent agreement with paleointensity measurements.

\section{Results}

Electrical conductivity. Our results show that the electrical conductivity of silicate liquid exceeds $10,000 \mathrm{~S} / \mathrm{m}$ at the conditions of the basal magma ocean (Fig. 1). The electrical conductivity increases with increasing temperature and decreases with increasing pressure. Magnetism reduces the electrical conductivity by $10 \%$. The primary charge carriers are electrons: the electronic contribution to $\sigma$ exceeds the ionic contribution by more than an order of magnitude. This behavior contrasts with experimental results at low pressure and temperature where ions are the dominant charge carriers ${ }^{8}$.

We can understand the electrical conductivity of silicate liquid by comparing with simpler systems and by examining the electronic density of states (Fig. 2). The electrical conductivity of the silicate liquid is intermediate to that of $\mathrm{SiO}_{2}$ liquid ${ }^{11,14,15}$ and $(\mathrm{Mg}, \mathrm{Fe}) \mathrm{O}$ liquid ${ }^{10}$ over the range of temperature and pressure that we have investigated. The conductivity of the silicate liquid is greater than that of $\mathrm{SiO}_{2}$ because of the role of iron. Iron $3 d$ states contribute to the density of states at the Fermi level and increase the conductivity compared with the iron-free system. The role of

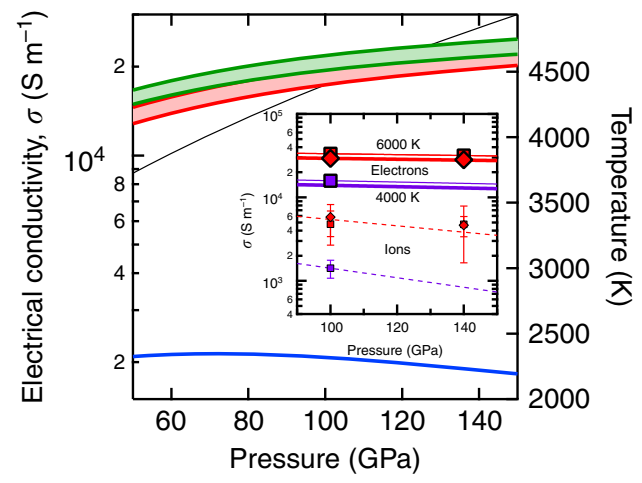

Fig. 1 Electrical conductivity. The electronic $\sigma_{\mathrm{el}}$ (red), ionic $\sigma_{\text {ion }}$ (blue), and total $\sigma_{\text {total }}=\sigma_{\mathrm{el}}+\sigma_{\text {ion }}$ (green) electrical conductivity along the magma ocean isentrope ${ }^{37}$ (black) for low-spin (top of range shown) and high-spin (bottom of range) results. Along the magma ocean isentrope, the electrical conductivity increases with depth because the effects of increasing temperature outweigh the effects of increasing pressure. Inset. The electrical conductivity of the bulk silicate Earth liquid from our simulations, including the electronic (large symbols) and ionic (small symbols with $1 \sigma$ uncertanties) contributions at $6000 \mathrm{~K}$ (red) and $4000 \mathrm{~K}$ (purple) in spinpolarized (diamonds) and non-spin-polarized (squares) calculations. Lines are best fits to the form $\sigma=\sigma_{0} T^{-1} \exp \left[-\left(E^{*}+P V^{*}\right) / R T\right]$ for the electronic non-spin-polarized results $\left(\sigma_{0}=1.994 \mathrm{e} 9 \mathrm{~S} / \mathrm{m}, E^{\star}=108.6 \mathrm{~kJ} / \mathrm{mol}, V^{\star}=\right.$ $0.0611 \mathrm{~cm}^{3} / \mathrm{mol}$, light solid lines) and shifted uniformly downward to account for spin-polarization $\left(\sigma_{0}=1.754 \mathrm{e} 9 \mathrm{~S} / \mathrm{m}\right.$, bold solid lines), and to the ionic contribution $\left(\sigma_{0}=1.0811 \mathrm{e} 9 \mathrm{~S} / \mathrm{m}, E^{\star}=131.0 \mathrm{~kJ} / \mathrm{mol}, V^{\star}=\right.$ $0.437 \mathrm{~cm}^{3} / \mathrm{mol}$, dashed lines).

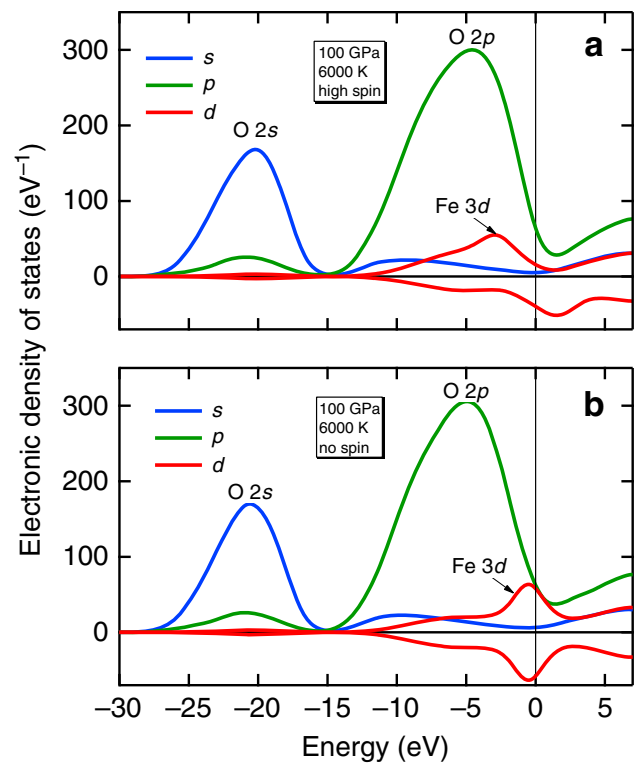

Fig. 2 Electronic density of states in the silicate liquid. At $100 \mathrm{GPa}$ and $6000 \mathrm{~K}$ in spin-polarized (a) and non-spin-polarized (b) states. Contributions from s- (blue), $p$ - (green) and $d$-like (red) states are shown separately and the dominant atomic contributions to each are indicated. The vertical black line is the Fermi level.

iron also explains why the temperature dependence of the electrical conductivity in the silicate liquid is less than that of $\mathrm{SiO}_{2}$ liquid (the conductivity in silica falls precipitously on cooling and drops ten times from 6000 to $4000 \mathrm{~K}$, whereas in the silicate liquid, it decreases only twofold): the highly localized iron $3 d$ states are less sensitive to their chemical environment than the $\mathrm{O}$ 
$2 p$ states that also contribute to conduction and their contribution to the density of states at the Fermi level is much less sensitive to temperature. The conductivity of the silicate liquid is less than that of $\left(\mathrm{Mg}_{0.75} \mathrm{Fe}_{0.25}\right) \mathrm{O}$ because the iron concentration is less in the silicate liquid $(\mathrm{Fe} /(\mathrm{Fe}+\mathrm{Mg})=0.11)$. The electronic density of states also explains why the conductivity of the spin-polarized system is slightly less than that of the non-spin-polarized system: magnetism splits up- and down-spin iron $3 d$ states, reducing the density of states at the Fermi level. While there are no experimental measurements of the electrical conductivity of silicate liquids at basal magma ocean conditions, we have shown that our approach yields results that are in good agreement with available experimental data at high pressure including the optical reflectivity of silica liquid and the electrical conductivity of crystalline $(\mathrm{Mg}, \mathrm{Fe}) \mathrm{O}^{10,11}$.

Magnetic field. To quantify magnetic field production, we combine our results for the electrical conductivity with a model of the thermal evolution of the basal magma ocean. The thermal evolution model is identical to that examined previously ${ }^{16}$, except that we have replaced the previously assumed value of the entropy of melting with the value determined from first principles molecular dynamics simulations (see Methods). This model yields the thickness of the magma ocean $L$ and heat flux out of the magma ocean $q$, which we combine with our values of the electrical conductivity to estimate the magnetic Reynolds number $R_{\mathrm{m}}=\left(L q / \rho H_{\mathrm{T}}\right)^{1 / 3} L \mu_{0} \sigma$ where $\rho$ is the mean density of the magma ocean and $H_{\mathrm{T}}$ is the thermal scale height. We have assumed the mixing length scaling for the flow velocity ${ }^{17}$. We estimate the magnetic field strength at the top of the basal magma ocean according to the scaling derived from numerical simulations ${ }^{17} B_{\mathrm{M}}=0.63 \rho^{1 / 3}\left(\mathrm{Lq} / \mathrm{H}_{\mathrm{T}}\right)^{2 / 3}$, where we have assumed that viscous dissipation is negligible because the ratio of viscous to magnetic diffusivity, the magnetic Prandtl number $P_{m}=$ $\mu_{0} \sigma \eta / \rho=10^{-7}$ is small, where $\eta=0.1 \mathrm{~Pa}$ is the viscosity of the basal magma ocean from previous first principles simulations ${ }^{18}$. Finally, we estimate the field strength at the surface $B_{\mathrm{S}}=\frac{1}{7} B_{\mathrm{M}}\left(r_{\mathrm{M}} / r_{\mathrm{E}}\right)^{3}$, where the pre-factor accounts for the proportion of the field strength in the dipole component, and the cubic dependence on radius accounts for the upward continuation of the dipole component to the surface: $r_{M}$ is the radius at the top of the basal magma ocean and $r_{\mathrm{E}}$ is the radius of the Earth ${ }^{6}$.

Our results show that a basal magma ocean is likely to have produced a magnetic field (Fig. 3). The magnetic field strength is very similar to that observed in the geologic record of the first few billion years of Earth's history. Moreover, the magnetic Reynolds number is sufficiently large to allow for a silicate dynamo in the early Earth. Although the basal magma ocean suppresses core convection, the core is likely to have strong horizontal flows that produce toroidal fields from the poloidal field generated by the basal magma ocean, reinforcing the silicate dynamo. The importance of this effect for the silicate dynamo is uncertain. Ziegler and Stegman ${ }^{19}$ suggested as the criterion for magnetic field generation the following dynamo number $D=\left(R_{\mathrm{m}} R_{\text {mcore }}\right)^{1 / 2}>100$, where $R_{\text {mcore }}=3500$ is the magnetic Reynolds number of the core computed assuming that the horizontal flow velocity is similar to that of the core today. According to the dynamo number criterion, the silicate dynamo may operate throughout the Archean (Fig. 3). Alternative velocity scalings also yield $D>100$ (see Methods). It is likely that the basal magma ocean became more iron-rich as it crystallized and that this iron enrichment increased the electrical conductivity. Our results for $R_{\mathrm{m}}$ and $D$ may therefore be underestimates. We have
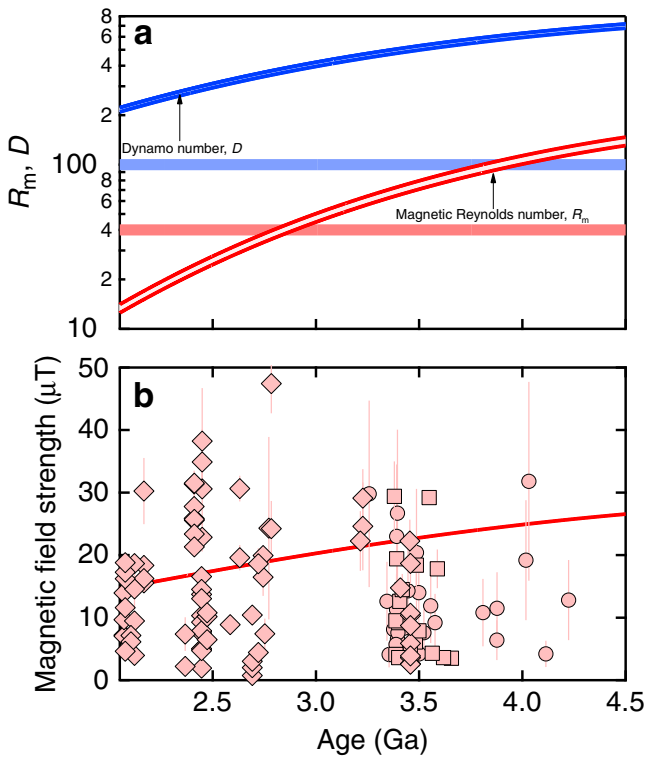

Fig. 3 Magnetic Reynolds number and magnetic field strength. a The magnetic Reynolds number (red) and Dynamo number (blue) computed from our electrical conductivity results and thermal evolution model with shading indicating the difference between low-spin (top of range) and highspin (bottom of range) results compared with the respective critical values of $R_{\mathrm{m}}=40$ and $D=100$ (thick horizontal lines). b Magnetic field strength computed from our model (red line) compared with paleomagnetic data: diamonds from the PINT database 40 , and circles (Thellier-Coe method) and squares ( $565 \mathrm{C}$ method) from zircons ${ }^{41}$. We note that the evidence of a field prior to the oldest whole-rock PINT data at $3.45 \mathrm{Ga}$ relies on the single-crystal zircon results, which have been questioned as not deriving from a primary magnetic carrier 42 .

not attempted a more quantitative treatment of the effects of iron enrichment because liquid-crystal iron partitioning and the magnitude of the effect of iron enrichment on the electrical conductivity of silicate liquid at basal magma ocean conditions are uncertain.

\section{Discussion}

The Earth's magnetic field may have transitioned from being produced by the basal magma ocean to being produced by the core near the end of the Archean: as the magma ocean cools, heat flow out of the core increases to the point that core convection, and a core dynamo, is possible (Supplementary Fig. 4b). Mechanisms have been proposed by which the core could produce a field earlier, including the presence of radioactive heat producing elements in the core $e^{3,20}$ and the precipitation of $\mathrm{Mg}^{4,21}$. However, recent experimental studies show that the solubility of $\mathrm{K}$ and $\mathrm{U}$ in the core is too small to power a dynamo 22,23 . Moreover, a recent reanalysis of experimental data shows that the temperature dependence of $\mathrm{Mg}$ precipitation, and thus the power produced, is a factor of six less than originally thought ${ }^{24}$ and may be less than that required to sustain a magnetic field ${ }^{25}$. The field produced by the silicate dynamo may have differed in geometry from the present day field and it may be possible to distinguish these unique characteristics in the geologic record. For example, there is some evidence from modeling magnetic field generation in other planetary bodies, including Uranus and Neptune ${ }^{26}$, that shallow layers, like the basal magma ocean, produce a less dipolar field than the strongly dipolar field of the present Earth. Many super-Earth exoplanets may have extensive magma oceans because of the greater amount of accretional energy stored in larger mass planets ${ }^{27}$, strong stellar 
irradiation, or the insulation of overlying gaseous envelopes ${ }^{28}$. Silicate dynamos may therefore be common in the universe.

\section{Methods}

System. Our system consists of 1129 atoms of seven different elements, with relative proportions chosen to closely match the six most abundant oxide components of the bulk silicate Earth (Supplementary Figs. 1 and 2, Supplementary Table 1).

Molecular dynamics simulations. Our molecular dynamics simulations are based on density functional theory in the PBEsol approximation ${ }^{29}$, combined with the $+U$ method $^{30}$ with $U-J=2.5 \mathrm{eV}$ as in our previous work ${ }^{10,31,32}$. We use the projector augmented wave $\operatorname{method}^{33}$, as implemented in VASP ${ }^{34}$. The core radii and number of electrons treated as valence for each element are listed in Supplementary Table 1. Born-Oppenheimer simulations are performed in the canonical ensemble using the Nosé-Hoover thermostat and run for 6-10 ps with $1 \mathrm{fs}$ time step. We perform spin-polarized simulations in which the difference in the number of up-spin and down-spin electrons is fixed to the high spin value (four times the number of Fe atoms) and also non-spin-polarized calculations. We assume thermal equilibrium between ions and electrons via the Mermin functional ${ }^{35}$. Sampling the Brillouin zone at the Gamma point and a basis-set energy cutoff of $500 \mathrm{eV}$ were found to be sufficient to converge energy and pressure to within $2 \mathrm{meV} /$ atom and $0.2 \mathrm{GPa}$, respectively. Our previous studies ${ }^{10,11}$ indicate that our simulations are well converged with respect to the number of atoms: in the case of $\mathrm{SiO}_{2}$, simulations with 96 and 144 atoms did not differ significantly in the value of $\sigma$, while in the case of $(\mathrm{Mg}, \mathrm{Fe}) \mathrm{O}$, differences in $\sigma$ between simulations containing 128,256 , and 512 atoms were less than $10 \%$.

Electronic conductivity and electronic density of states. We compute the electronic conductivity via the Kubo-Greenwood formula ${ }^{12}$ as implemented in VASP from a series of at least 10 uncorrelated snapshots at each volume-temperature condition (Supplementary Fig. 3). The frequency dependent electronic conductivity is

$$
\sigma_{\mathrm{el}}(\omega)=\frac{2 \pi e^{2} \hbar^{2}}{3 m^{2} \omega \Omega} \sum_{\vec{k}} \sum_{i, j}\left[f\left(\varepsilon_{j}, \vec{k}\right)-f\left(\varepsilon_{i}, \vec{k}\right)\right]\left|\left\langle\psi_{i, \vec{k}}\right\rangle\right| \nabla\left|\left\langle\psi_{j, \vec{k}}\right\rangle\right|^{2} \delta\left(\varepsilon_{i}-\varepsilon_{j}-\hbar \omega\right),
$$

where the sums are over the Brillouin zone and pairs of states, respectively, $f$ is the Fermi occupation, $\psi$ is the wavefunction, $\varepsilon$ is the eigenvalue, $\omega$ is the frequency, and $\Omega$ is the volume of the simulation cell. In our computations, the $\delta$ function is replaced by a Gaussian of width $\Delta$ given by the average spacing between eigenvalues weighted by the corresponding change in the Fermi function ${ }^{36}$. As the behavior of Eq. 1 becomes unphysical for $\hbar \omega<\Delta$, we find the DC conductivity by linearly extrapolating to zero frequency.

We found that a $1 \times 1 \times 1 \mathrm{k}$-point mesh and 7200 electronic bands were sufficient to yield converged values of the electronic conductivity and the electronic density of states by performing calculations at doubled Brillouin zone sampling $(2 \times 2 \times 2$ k-point mesh $)$ and a larger number of electronic bands $(10,200)$. We compute the electronic density of states by averaging over 10 snapshots well separated in time from the molecular dynamics trajectories. We use the energy derivative of the Fermi-Dirac function with temperature equal to the ionic temperature to smooth the density of states.

Ionic conductivity. We compute the ionic part of the electrical conductivity in the DC limit from the electric current autocorrelation function ${ }^{13}$

$$
J(t)=\sum_{i, j} z_{i} z_{j}\left\langle\vec{u}_{i}\left(t+t_{0}\right) \cdot \vec{u}_{j}\left(t_{0}\right)\right\rangle,
$$

as the integral

$$
\sigma_{\text {ion }}=\frac{e^{2}}{3 k T \Omega} \int J(t) \mathrm{d} t .
$$

where $z_{i}$ and $\vec{u}_{i}$ are the Bader charge and velocity of ion $i$, respectively, and the angle brackets indicate an average over time origins $t_{0}$. The Bader charges of the ions computed in our simulation are listed in Supplementary Table 1.

Thermal evolution of the basal magma ocean. We solve the coupled system of equations 16 :

$$
\begin{gathered}
4 \pi a^{2} k \frac{T_{\mathrm{L}}-T_{\mathrm{M}}}{\delta}=-\left(M_{\mathrm{m}} c_{\mathrm{m}}+M_{\mathrm{c}} c_{\mathrm{c}}\right) \frac{\mathrm{d} T_{\mathrm{L}}}{\mathrm{d} t}+H(t)-4 \pi a^{2} \rho \Delta S T_{\mathrm{L}} \frac{\mathrm{d} a}{\mathrm{~d} t}, \\
T_{L}=T_{A}-\left(T_{A}-T_{B}\right) \xi, \\
\frac{\mathrm{d} \xi_{L}}{\mathrm{~d} t}=-\frac{3 a^{2} \Delta \xi}{a^{3}-b^{3}} \frac{\mathrm{d} a}{\mathrm{~d} t},
\end{gathered}
$$

where the first equation expresses the relationship between the time evolution of the outer radius of the basal magma ocean $a$, and its temperature $T_{\mathrm{L}}$ where $k$ is the thermal conductivity, $T_{\mathrm{M}}$ is the temperature of the overlying solid mantle, $\delta$ is the thickness of thermal boundary layer at the base of the overlying mantle, $M$ and $c$ are the mass and isobaric specific heat, respectively of the basal magma ocean (subscript $m$ ) and core (subscript $c$ ), $H$ is the radioactive heat production, $\rho$ is the density of the basal magma ocean, and $\Delta S$ is the entropy change on freezing. The inner radius of the basal magma ocean $b$ is assumed to be the core-mantle boundary. The term on the left-hand side is the total heat flux out of the basal magma ocean. On the right-hand side we have contributions from, respectively, cooling of the basal magma ocean and the core, radioactive heat production, and latent heat of freezing. The second equation expresses the idealized phase diagram defined by a linear dependence of the liquidus temperature on the mass fraction of the dense component $\xi$, where $T_{A}$ and $T_{B}$ are the melting temperatures of the endmember light $(A)$ and dense $(B)$ components. The last equation is a statement of mass balance where $\Delta \xi=\xi_{\mathrm{L}}-\xi_{\mathrm{S}}$ is the enrichment of the liquid in the dense component. The model does not include inner core growth, nor radioactive heating, nor Mg-exsolution in the core.

We solve the equations with a fourth-order Runge-Kutta method and adopt values of all parameters identical to those assumed in ref. ${ }^{16}$, except for the entropy of melting, which we take from our previous simulations $\Delta S=652 \mathrm{~J} / \mathrm{kg} / \mathrm{K}^{37}$ (Supplementary Table 2). The value of $\delta$ is chosen to yield a present day temperature at the core-mantle boundary of $4000 \mathrm{~K}$.

Magnetic Reynolds number. We compute the magnetic Reynolds number of the basal magma ocean as

$$
R_{\mathrm{m}}=\mu_{0} v L \sigma,
$$

where $\mu_{0}$ is the permeability of free space, $L$ is the thickness of the basal magma ocean, $v$ is the flow velocity, and $\sigma$ is the electrical conductivity. We take the value of $L=a-b$ from the thermal evolution model (Supplementary Fig. 4). The value of $\sigma$ is the total electrical conductivity computed at the base of the basal magma ocean and the temperature $T_{\mathrm{L}}$ from the thermal evolution model (Supplementary Fig. 4) and the equations for the temperature dependence of $\sigma_{\mathrm{el}}$ and $\sigma_{\mathrm{ion}}$ given in the caption to Fig. 1 in the main text.

To estimate the flow velocity, we use the scaling recommended by numerical simulations ${ }^{17}$ from mixing length theory

$$
v=\left(\frac{L q}{\rho H_{\mathrm{T}}}\right)^{1 / 3},
$$

where $q$ is the total heat flow out of top of the basal magma ocean (Supplementary Fig. 4b), $\rho$ is the density of the magma ocean, and $H_{\mathrm{T}}$ is the thermal scale height, which we find from our previous simulation results ${ }^{37}$ to be $H_{\mathrm{T}}=7622 \mathrm{~km}$.

Following ${ }^{19}$, we also examine alternative scalings for the velocity, including those based on a balance between Coriolis, inertial, and Archimedean (buoyancy) forces: CIA

$$
v=\left(\frac{q}{\rho H_{\mathrm{T}}}\right)^{2 / 5}\left(\frac{L}{\Omega}\right)^{1 / 5}
$$

and from a balance between the buoyancy and Lorentz forces: MAC

$$
v=\left(\frac{q}{\Omega \rho H_{\mathrm{T}}}\right)^{1 / 2},
$$

where $\Omega$ is the rotation rate.

We also estimate the possible effects of coupling of the magnetic field produced by the basal magma ocean with the underlying, non-convecting core. Although the basal magma ocean suppresses core convection, the core is likely to have strong horizontal flows that generate toroidal fields from the poloidal field produced by the basal magma ocean, reinforcing the silicate dynamo. Ziegler and Stegman ${ }^{19}$ suggested as the criterion for magnetic field generation the following dynamo number $D$ : the geometric mean of the magnetic Reynolds numbers of the basal magma ocean and the core

$$
D=\left(R_{\mathrm{m}} R_{m \text { core }}\right)^{1 / 2},
$$

where $R_{\text {mcore }}=3500$ is the magnetic Reynolds number of the core computed assuming that the horizontal flow velocity is similar to that of the core today, and $D>100$ in order to produce a magnetic field. Following Ziegler and Stegman, we have estimated $R_{\text {mcore }}$ by assuming that the horizontal flow velocity is similar to that of the core today $\left(v=5 \times 10^{-4} \mathrm{~m} / \mathrm{s}\right)^{38}$, the conductivity, $\sigma=1.5 \times 10^{6} \mathrm{~S} / \mathrm{m}^{39}$, and $L=3500 \mathrm{~km}$.

\section{Data availability}

Raw simulation results are available from the corresponding author on request.

\section{Code availability}

VASP is widely used proprietary software. 
Received: 25 August 2019; Accepted: 29 January 2020;

Published online: 25 February 2020

\section{References}

1. Tarduno, J. A. et al. Geodynamo, solar wind, and magnetopause 3.4 to 3.45 billion years ago. Science 327, 1238-1240 (2010).

2. Labrosse, S. Thermal and magnetic evolution of the Earth's core. Phys. Earth Planet. Interiors 140, 127-143 (2003).

3. Nimmo, F., Price, G. D., Brodholt, J. \& Gubbins, D. The influence of potassium on core and geodynamo evolution. Geophys. J. Int. 156, 363-376 (2004).

4. O'Rourke, J. G. \& Stevenson, D. J. Powering Earth's dynamo with magnesium precipitation from the core. Nature 529, 387-389 (2016).

5. Stevenson, D. J. Planetary magnetic fields. 1-11, https://doi.org/10.1016/ S0012-821X(02)01126-3 (2003).

6. Christensen, U. R. \& Aubert, J. Scaling properties of convection-driven dynamos in rotating spherical shells and application to planetary magnetic fields. Geophys. J. Int. 166, 97-114 (2006).

7. Leorat, J. \& Nore, C. Interplay between experimental and numerical approaches in the fluid dynamo problem. C. R. Phys. 9, 741-748 (2008).

8. Ni, H. W., Hui, H. J. \& Steinle-Neumann, G. Transport properties of silicate melts. Rev. Geophys. 53, 715-744 (2015).

9. Pommier, A., Leinenweber, K. \& Tasaka, M. Experimental investigation of the electrical behavior of olivine during partial melting under pressure and application to the lunar mantle. Earth Planet. Sci. Lett. 425, 242-255 (2015).

10. Holmstrom, E., Stixrude, L., Scipioni, R. \& Foster, A. S. Electronic conductivity of solid and liquid ( $\mathrm{Mg}, \mathrm{Fe}) \mathrm{O}$ computed from first principles. Earth Planet. Sci. Lett. 490, 11-19 (2018).

11. Scipioni, R., Stixrude, L. \& Desjarlais, M. P. Electrical conductivity of $\mathrm{SiO}_{2}$ at extreme conditions and planetary dynamos. Proc. Natl. Acad. Sci. USA 114 9009-9013 (2017).

12. Desjarlais, M. P., Kress, J. D. \& Collins, L. A. Electrical conductivity for warm, dense aluminum plasmas and liquids. Phys. Rev. E 66, 025401(R) (2002).

13. Hansen, J. P. \& McDonald, I. R. Statistical-mechanics of dense ionized matter. 4. Density10 and charge fluctuations in a simple molten-salt. Phys. Rev. A 11, 2111-2123 (1975).

14. Soubiran, F. \& Militzer, B. Electrical conductivity and magnetic dynamos in magma oceans of Super-Earths. Nat. Commun. 9, 7 (2018).

15. Qi, T. T., Millot, M., Kraus, R. G., Root, S. \& Hamel, S. Optical and transport properties of dense liquid silica. Phys. Plasmas 22, 10 (2015).

16. Labrosse, S., Hernlund, J. W. \& Coltice, N. A crystallizing dense magma ocean at the base of the Earth's mantle. Nature 450, 866-869 (2007).

17. Christensen, U. R. Dynamo scaling laws and applications to the planets. Space Sci. Rev. 152, 565-590 (2010).

18. Karki, B. B. \& Stixrude, L. P. Viscosity of $\mathrm{MgSiO} 3$ liquid at Earth's mantle conditions: implications for an early magma ocean. Science 328, 740-742 (2010).

19. Ziegler, L. B. \& Stegman, D. R. Implications of a long-lived basal magma ocean in generating Earth's ancient magnetic field. Geochem. Geophys. Geosyst. 14, 4735-4742 (2013).

20. Bukowinski, M. S. T. The effect of pressure on the physics and chemistry of potassium. Geophys. Res. Lett. 3, 491-494 (1976).

21. Badro, J., Siebert, J. \& Nimmo, F. An early geodynamo driven by exsolution of mantle components from Earth's core. Nature 536, 326-32 (2016).

22. Blanchard, I., Siebert, J., Borensztajn, S. \& Badro, J. The solubility of heatproducing elements in Earth's core. Geochem. Perspect. Lett. 5, 1-5 (2017).

23. Chidester, B. A., Rahman, Z., Righter, K. \& Campbell, A. J. Metal-silicate partitioning of $\mathrm{U}$ : implications for the heat budget of the core and evidence for reduced U in the mantle. Geochim. Cosmochim. Acta 199, 1-12 (2017).

24. Badro, J. et al. Magnesium partitioning between earth's mantle and core and its potential to drive an early exsolution geodynamo. Geophys. Res. Lett. 45, 13240-13248 (2018).

25. O'Rourke, J. G., Korenaga, J. \& Stevenson, D. J. Thermal evolution of Earth with magnesium precipitation in the core. Earth Planet. Sci. Lett. 458, 263-272 (2017).

26. Stanley, S. \& Bloxham, J. Numerical dynamo models of Uranus' and Neptune's magnetic fields. Icarus 184, 556-572 (2006)

27. Stixrude, L. Melting in super-earths. Philos. Trans. R. Soc. A Math. Phys. Eng. Sci. 372, 20130076 (2014).

28. Ginzburg, S., Schlichting, H. E. \& Sari, R. Super-earth atmospheres: selfconsistent gas accretion and retention. Astrophys. J. 825, 12 (2016).

29. Perdew, J. P. et al. Restoring the density-gradient expansion for exchange in solids and surfaces. Phys. Rev. Lett. 100, 136406 (2008).

30. Dudarev, S. L., Botton, G. A., Savrasov, S. Y., Humphreys, C. J. \& Sutton, A. P. Electron-energy-loss spectra and the structural stability of nickel oxide: An LSDA+U study. Phys. Rev. B 57, 1505-1509 (1998).
31. Holmstrom, E. \& Stixrude, L. Spin crossover in liquid ( $\mathrm{Mg}, \mathrm{Fe}) \mathrm{O}$ at extreme conditions. Phys. Rev. B 93, 195142 (2016).

32. Holmstrom, E. \& Stixrude, L. Spin crossover in ferropericlase from firstprinciples molecular dynamics. Phys. Rev. Lett. 114, 117202 (2015).

33. Kresse, G. \& Joubert, D. From ultrasoft pseudopotentials to the projector augmented-wave method. Phys. Rev. B 59, 1758-1775 (1999).

34. Kresse, G. \& Furthmüller, J. Efficient iterative schemes for ab initio totalenergy calculations using a plane-wave basis set. Phys. Rev. B 54, 11169-11186 (1996).

35. Mermin, N. D. Thermal properties of the inhomogeneous electron gas. Phys. Rev. 137, A1441-A1443 (1965).

36. Pozzo, M., Desjarlais, M. P. \& Alfe, D. Electrical and thermal conductivity of liquid sodium from first-principles calculations. Phys. Rev. B 84, 054203 (2011)

37. Stixrude, L., de Koker, N., Sun, N., Mookherjee, M. \& Karki, B. B. Thermodynamics of silicate liquids in the deep Earth. Earth Planet. Sci. Lett. 278, 226-232 (2009).

38. Holme, R. in Treatise on Geophysics Vol. 8 (ed. Schubert, G.) 107-130 (Elsevier, 2007).

39. Pozzo, M., Davies, C., Gubbins, D. \& Alfé, D. Thermal and electrical conductivity of iron at Earth's core conditions. Nature 485, 355-U399 (2012).

40. Biggin, A. J., Strik, G. \& Langereis, C. G. The intensity of the geomagnetic field in the late-Archaean: new measurements and an analysis of the updated IAGA palaeointensity database. Earth Planets Space 61, 9-22 (2009).

41. Tarduno, J. A., Cottrell, R. D., Davis, W. J., Nimmo, F. \& Bono, R. K. A Hadean to Paleoarchean geodynamo recorded by single zircon crystals. Science 349, 521-524 (2015)

42. Tang, F. Z. et al. Secondary magnetite in ancient zircon precludes analysis of a Hadean geodynamo. Proc. Natl. Acad. Sci. USA 116, 407-412 (2019).

\section{Acknowledgements}

This project is supported by the European Research Council under Advanced Grant 291432 MoltenEarth (FP7) and by the National Science Foundation (EAR-1853388). Calculations were carried out using the IRIDIS cluster partly owned by University College London, ARCHER of the UK National High-Performance Computing Service, and MARENOSTRUM at the Barcelona Supercomputing Center, Spain of the PRACE Consortium. We thank J. Aurnou for helpful comments on the manuscript.

\section{Author contributions}

L.S. and R.S. designed and performed the research, and analyzed the data. M.P.D. provided analytical tools. L.S., R.S., and M.P.D. wrote the paper.

\section{Competing interests}

The authors declare no competing interests.

\section{Additional information}

Supplementary information is available for this paper at https://doi.org/10.1038/s41467020-14773-4.

Correspondence and requests for materials should be addressed to L.S.

Peer review information Nature Communications thanks Ulrich Christensen, Joseph O'Rourke and the other, anonymous, reviewer(s) for their contribution to the peer review of this work.

Reprints and permission information is available at http://www.nature.com/reprints

Publisher's note Springer Nature remains neutral with regard to jurisdictional claims in published maps and institutional affiliations.

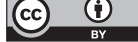

Open Access This article is licensed under a Creative Commons Attribution 4.0 International License, which permits use, sharing, adaptation, distribution and reproduction in any medium or format, as long as you give appropriate credit to the original author(s) and the source, provide a link to the Creative Commons license, and indicate if changes were made. The images or other third party material in this article are included in the article's Creative Commons license, unless indicated otherwise in a credit line to the material. If material is not included in the article's Creative Commons license and your intended use is not permitted by statutory regulation or exceeds the permitted use, you will need to obtain permission directly from the copyright holder. To view a copy of this license, visit http://creativecommons.org/ licenses/by/4.0/.

(C) The Author(s) 2020 